\title{
Electron scattering from 1-butanol at intermediate impact energies: Total cross sections
}

Cite as: J. Chem. Phys. 150, 194307 (2019); https://doi.org/10.1063/1.5096211

Submitted: 15 March 2019 . Accepted: 28 April 2019 . Published Online: 20 May 2019

M. Gomes (D, D. G. M. da Silva, A. C. P. Fernandes, S. Chosh, W. A. D. Pires, D. B. Jones (D), F. Blanco (D), C. García (D), M. J. Brunger (D), and M. C. A. Lopes (D)

\section{ARTICLES YOU MAY BE INTERESTED IN}

Trajectory-adjusted electronic zero point energy in classical Meyer-Miller vibronic dynamics: Symmetrical quasiclassical application to photodissociation

The Journal of Chemical Physics 150, 194110 (2019); https://doi.org/10.1063/1.5094458

On the importance of onset times and multiple-wavelength analysis of photoluminescence decays

Journal of Applied Physics 125, 193103 (2019); https://doi.org/10.1063/1.5097065

High-resolution infrared spectroscopy of jet cooled trans-deuteroxycarbonyl (trans-DOCO) radical

The Journal of Chemical Physics 150, 194304 (2019); https://doi.org/10.1063/1.5092599
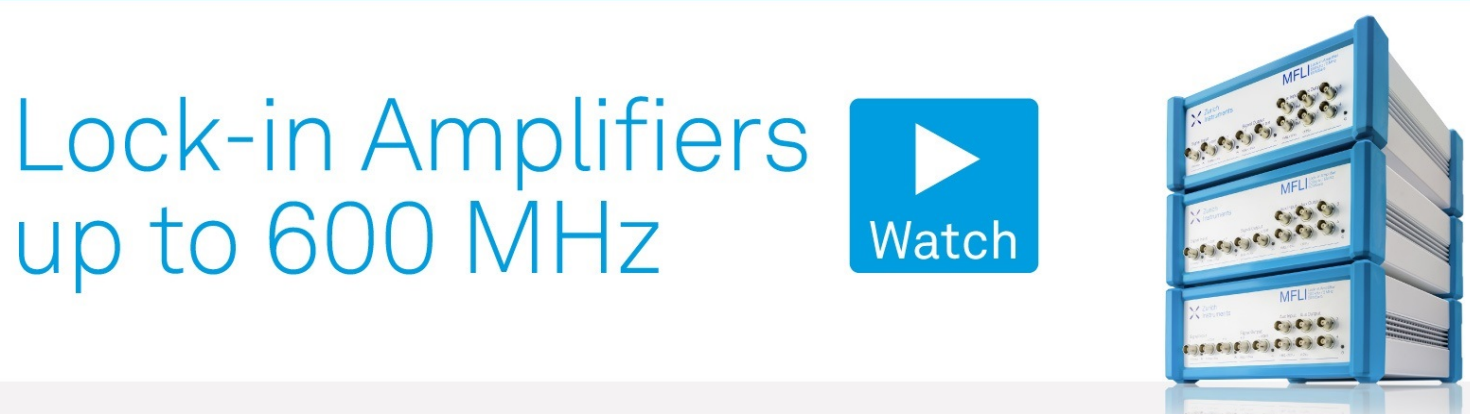


\title{
Electron scattering from 1-butanol at intermediate impact energies: Total cross sections
}

\author{
Cite as: J. Chem. Phys. 150, 194307 (2019); doi: 10.1063/1.5096211 \\ Submitted: 15 March 2019 - Accepted: 28 April 2019 • \\ Published Online: 20 May 2019
}

\author{
M. Gomes, ${ }^{1}$ (D) D. G. M. da Silva, ${ }^{1}$ A. C. P. Fernandes, ${ }^{1}$ S. Ghosh, ${ }^{1}$ W. A. D. Pires, ${ }^{1}$ D. B. Jones, \\ F. Blanco, \\ G. García, ${ }^{4}$ \\ M. J. Brunger, ${ }^{2}$ \\ and M. C. A. Lopes
}

\begin{abstract}
AFFILIATIONS
${ }^{1}$ Departamento de Física, Universidade Federal de Juiz de Fora, Juiz de Fora, MC 36036-900, Brazil

${ }^{2}$ College of Science and Engineering, Flinders University, GPO Box 2100, Adelaide, SA 5001, Australia

${ }^{3}$ Departamento de Física Atómica, Molecular y Nuclear, Universidad Complutense de Madrid, 28040 Madrid, Spain

${ }^{4}$ Instituto de Física Fundamental, CSIC, Serano 113-bis, 28006 Madrid, Spain
\end{abstract}

a) Author to whom correspondence should be addressed: michael.brunger@flinders.edu.au

\begin{abstract}
We report experimental measurements of the absolute total cross sections (TCSs) for electron scattering from 1-butanol at impact energies in the range $80-400 \mathrm{eV}$. Those measurements were conducted by considering the attenuation of a collimated electron beam, at a given energy, through a gas cell containing 1-butanol, at a given pressure, and through application of the Beer-Lambert law to derive the required TCS. We also report theoretical results using the Independent-Atom Model with Screening Corrected Additivity Rule and Interference approach. Those results include the TCS, the elastic integral cross section (ICS), the ionization total ICS, and the sum over all excitation process ICSs with agreement at the TCS level between our measured and calculated results being encouraging.
\end{abstract}

Published under license by AIP Publishing. https://doi.org/10.1063/1.5096211

\section{INTRODUCTION}

We have recently published two papers ${ }^{1,2}$ that looked at the mass spectra, appearance energies, partial ionization integral cross sections (ICSs), and the total ionization cross section (TICS) for electron impact ionization and dissociative ionization in 1-butanol $\left(\mathrm{C}_{4} \mathrm{H}_{9} \mathrm{OH}\right)$. Note that those earlier results were measured on a completely different apparatus to what we employ here (see later) and are different physical entities to the total cross sections (TCSs) we report here. As a part of those studies, we discussed in detail why 1-butanol might make a cleaner and more economical fuel, ${ }^{3}$ compared to petrol, and that in order to achieve that aim, modeling of its autoignition behavior needed to be undertaken. In general, whether it be to better comprehend atmospheric phenomena, ${ }^{4,5}$ low temperature plasmas, ${ }^{6}$ or charged-particle track behavior, ${ }^{7-10}$ Tanaka et al. ${ }^{1}$ noted that it was in part necessary to have complete and comprehensive cross section databases (see, e.g., Ref. 12) in order to accurately model or simulate those phenomena. This is also true for simulating the specific ignition properties of 1-butanol. Aside from the aforementioned papers from our group, ${ }^{1,2}$ Hudson et al. ${ }^{13}$ have reported absolute TICS for electron scattering from 1-butanol in the energy range 16-207 eV. In addition, Khakoo et al. ${ }^{14}$ reported extensive results, both experimental and theoretical (using the Schwinger multichannel method), for elastic differential, integral, and momentum transfer cross sections (MTCSs). The experimental differential cross section (DCS) measurements were conducted in the energy range $1-100 \mathrm{eV}$ and over the scattered electron angular range $5^{\circ}-130^{\circ}$. As a consequence, to derive their measured elastic integral cross sections (ICSs) and momentum transfer cross sections (MTCSs), an extrapolation of their DCS to $0^{\circ}$ and $180^{\circ}$ was needed before the requisite integration could be made. Finally, we note the dissociative electron attachment (DEA) work of Ibănescu and Allan ${ }^{15}$ that indicated resonance enhanced ion yields for the $\mathrm{OH}^{-}$and $(\mathrm{M}-1)^{-}$ anions. Unfortunately, those latter results ${ }^{15}$ were only relative so that the importance of this scattering channel is yet to be elucidated. Thus, there remains a lack of absolute cross section data in relation 
to electron scattering from 1-butanol, which the present investigation seeks to address in part. This facet forms one rationale for the present study.

Total cross sections have in more recent times been somewhat undervalued ${ }^{16}$ as in the so-called hierarchy of cross sections (so named in terms of how much physical information they convey about the scattering dynamics in question), they sit at the bottom of the table. Nonetheless, even though they do not discriminate between the various open scattering channels at a given energy, nor do they provide any angular scattering information, they still fulfill several vital roles. ${ }^{17}$ Namely, as the TCS is the upper limit for the sum of the various ICSs for all open processes at a given energy, it is ideal for assessing the validity of cross sections for individual scattering processes (sum conservation). These selfconsistency checks are particularly important as TCS can typically be obtained with much higher accuracy ${ }^{16}$ than higher-level cross sections and so provide a more robust assessment of the available data. The TCS also plays a vital role for scientists in the modeling and simulation communities who are attempting to construct databases, as they can often be used, in conjunction with other ICSs, to estimate the cross section data for specific scattering processes that are unavailable. In addition and perhaps this is not so well appreciated in the scattering community, they provide a direct link to parameters like the stopping power [see Eq. (19) of Ref. 18] which are fundamental in describing charged-particle track behavior in atoms and molecules. As a consequence, we believe the measurements and calculations of TCSs that we report later for 1-butanol remain topical and important in many areas of science and in understanding and characterizing various applications including technology. ${ }^{19,2}$

It is well-known ${ }^{21-23}$ that the valence electronic properties of a molecule, such as its permanent dipole moment $(\mu)$ and dipole polarizability $(\alpha)$, affect the scattering dynamics and therefore the scattering cross sections. Here, we are particularly interested in comparing our earlier measured results for methanol, ${ }^{24}$ ethanol, ${ }^{24}$ and 1-propanol ${ }^{25}$ with those we report here for 1-butanol. Each of these species are linear primary alcohols and all have permanent dipole moments in the narrow range $\mu=1.55 \mathrm{D}-1.70 \mathrm{D}{ }^{26,27}$ On the other hand, the dipole polarizabilities for these species are very different, with methanol having $\alpha=3.21 \AA^{3}$, , ethanol having $\alpha=4.92 \AA^{3}, 28$ 1-propanol having $\alpha=6.67 \AA^{3},{ }^{28}$ and 1-butanol having $\alpha=8.57 \AA^{3}{ }^{28}$ Thus, the comparison between our intermediate energy TCS for these species might well present us with an opportunity to try and elucidate the role of $\alpha$ in their scattering dynamics. This facet forms another rationale for this investigation.

The remainder of this paper is organized as follows: In Sec. II, we present details of our experimental apparatus and techniques, including a discussion of our measurement uncertainties, as well as details on our independent-atom model with screening corrected additivity rule and interference terms (IAM-SCAR+I) theoretical approach. Section III details our TCS results and a discussion of those results, and finally, in Sec. IV, we provide some conclusions from this study.

\section{EXPERIMENTAL AND THEORETICAL DETAILS}

We previously described our experimental apparatus and measurement techniques in some detail ${ }^{25}$ so that only a précis of that discussion, in order to make this paper self-contained, needs to be given here. The current apparatus consists of an electron gun, a gas cell, an electron energy analyzer composed of an array of decelerating electrostatic lenses and a cylindrical dispersive $127^{\circ}$ analyzer, and a Faraday cup. Note that the overall energy resolution we obtained with this apparatus was typically $0.7 \mathrm{eV}$ (FWHM). As the energy range of the present investigation is $80-400 \mathrm{eV}$, where no resonance scattering effects ${ }^{16}$ are expected, the current energy resolution will not affect our TCS data. This apparatus is housed inside a vacuum chamber enclosed by 5 layers of mu-metal, to shield against spurious magnetic fields, and is differentially pumped through 2 turbomolecular pumps. The first of those turbomolecular pumps has a pumping speed of $80 \mathrm{l} / \mathrm{s}$ and services the electron gun chamber in order to avoid possible changes in the electron-emitting filament when 1-butanol is introduced into the chamber. The second, with pumping speed $600 \mathrm{l} / \mathrm{s}$, services the scattering cell and analyzer region. ${ }^{2}$

Our apparatus utilizes an in-house designed electron gun and its associated control electronics, which, in principle, covers an energy range from 5 to $500 \mathrm{eV}$. The performance of this gun was typically very good, providing a suitable focus and collimation into the scattering cell that is necessary for the successful application of the linear transmission technique. ${ }^{24}$ The present scattering cell is a $40.0 \mathrm{~mm}$ long tube, which is bounded at its entrance and exit by two apertures of $2.0 \mathrm{~mm}$ diameter. The collimated electron beam passes through the scattering cell, and those electrons that traverse this cell are discriminated with the electrostatic lens and a $127^{\circ}$ cylindrical dispersive analyzer, which prevents electrons inelastically scattered in the forward direction being collected by the Faraday cup. The angular acceptance of the analyzer, defined by the solid angle formed by the analyzer aperture as seen from the center of the scattering cell, is $6.4 \times 10^{-4} \mathrm{sr}$. The temperature of the 1-butanol sample is measured using a mercury thermometer, to $\pm 1^{\circ} \mathrm{C}$, that is fixed on the external wall of the vacuum chamber. This follows as it was previously established ${ }^{24}$ that the temperature of the gas sample in the scattering cell was the same as that of the vacuum chamber. The 1-butanol gas is introduced into the chamber using a leak valve, while its pressure $P$ is monitored by a MKS Baratron. A schematic diagram of the present experimental configuration is given in Fig. 1.

All the present measurements were performed for an incident beam current of around $10^{-9} \mathrm{~A}$, where no dependence of the TCS upon current was exhibited. Note that the typical currents measured at the Faraday cup were $500 \mathrm{pA}$ with no gas in the cell and $400 \mathrm{pA}$ at maximum attenuation. The TCS, $\sigma(\mathrm{E})$, obtained through the linear attenuation technique, ${ }^{16}$ is based on the measurement of the attenuation of the collimated electron beam at a given energy (E) through a scattering cell containing the 1-butanol target at a given pressure $(P)$ and temperature $(T)$, which is given by the Beer-Lambert law

$$
I_{t}(E)=I_{0}(E) e^{-n L \sigma(E)},
$$

where $I_{0}(E)$ and $I_{\mathrm{t}}(E)$ are the intensity of the electron beam, as measured in the Faraday cup (see Fig. 1), before and after the gas (scattering) cell, respectively, $n$ is the average particle number density of 1-butanol, and $L$ is the path length of the electron beam through the gas cell. The TCS data are, in practice, determined by 


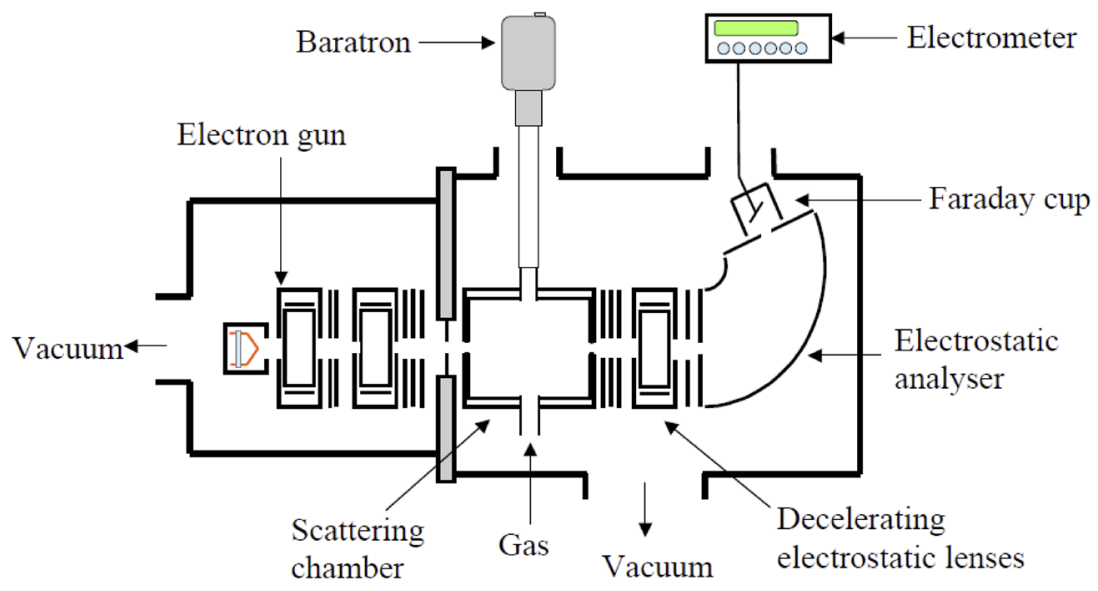

FIG. 1. Schematic diagram of the present experimental configuration.

measuring the transmitted intensities with and without gas in the cell; $n$ is taken to be equal to $P / k T$, using the ideal gas law, where $P$ is the pressure of the 1-butanol gaseous target measured with a Baratron in the molecular flow region, $k$ is Boltzmann's constant, and $T$ is the temperature in Kelvin. The pressure range in the scattering cell was maintained between 1 and 4 mTorr so that possible double scattering effects were minimized. With these precautions, the variation of $\ln \left(I_{t} / I_{0}\right)$ with $P$ is a straight line whose slope is proportional to the TCS. Similar to our work in 1-proponal, ${ }^{25}$ the current measurements were carried out for at least five runs, each run taking at least seven sets of values for $P, I_{0}$, and $I_{\mathrm{t}}$. An averaging procedure, with an associated calculation of the standard deviation, was subsequently applied to determine the TCS at a given energy.

The uncertainties on our TCS data were estimated by taking into account the statistical uncertainty, estimated in the range around $2 \%-12.8 \%$, fluctuations in the primary electron beam of $\sim 1.5 \%$, the accuracy of the pressure reading from the MKS Baratron of $\sim 2 \%$, and knowledge of the effective scattering length of the gas cell $(\sim 0.3 \%)$. In addition, we note that the stability of the flow of gas entering the gas cell varied by less than $1 \%$, while the temperature of the gas in the cell was measured to an accuracy of $0.5 \%$. Considering all these factors, the root square sum of the overall TCS uncertainties was in the range around 3.4\%-13.1\%. The bona fides of the present apparatus and our measurement procedures were thoroughly checked against previous results from a standard system such as argon. Here, we found, across the energy range $14-500 \mathrm{eV}$, excellent agreement ${ }^{25}$ between results taken with our apparatus and those from Wagenaar and de Heer ${ }^{29}$ and Szmythowski et al., ${ }^{30}$ for example.

1-butanol vapor was obtained from the volatile butanol liquid, sourced from Sigma-Aldrich, with a stated purity of $99.5 \%$. In addition, we degassed the sample using at least six freeze-pump-thaw cycles before it was admitted into the chamber using a needle valve (Granville-Phillips-203 variable leak valve ${ }^{31}$ ). The vapor pressure of 1-butanol in its storage container was calculated to be 5.32 Torr, using the Antoine equation. ${ }^{32}$ The butanol liquid was housed in a glass flask, vacuum coupled to the relevant leak valve by a glassto-metal seal, with a $1 / 4$ " Covar tube being used to send vapor into the gas feedline. Although we anticipate some condensation of 1-butanol in our gas lines, gas cell, and on other elements of the spectrometer, the present experiments were performed without baking. Hence, they were conducted at the laboratory temperature of around $22^{\circ} \mathrm{C}$, as maintained using conditioned air. There is no doubt that this decision not to bake, due to a lack of appropriate heating tapes etc., made the present measurements more of a challenge than would otherwise be the case and certainly contributed to the overall uncertainty on our TCS being a little higher than one might normally expect. $^{16,17}$

Finally, we note that the present results are uncorrected for the so-called "forward angle scattering effect." ${ }^{33}$ However, this effect, which results from a very forward angle elastically, and given our energy resolution rotationally, scattered electrons not being discriminated against in our detector system, is expected to be small in this case. ${ }^{33}$ This follows as such corrections are known to be typically smaller at the intermediate energies of this work, ${ }^{19,34}$ even for polar molecules such as 1-butanol, and given the very good angular resolution (less than $1^{\circ}$ ) of our present spectrometer. Indeed, we calculate that "forward angle scattering effect" to be less than $1.2 \%$ over the energy range of this study.

The IAM-SCAR+I approach has already been discussed many times, ${ }^{35,36}$ most recently by Traoré Dubuis et al. ${ }^{20}$ and so only a brief description is needed here. The basic premise of this method is that the molecule of interest is not considered as a single multicentre target but as an aggregate of the individual atoms. ${ }^{37}$ As a consequence, this paradigm effectively assumes that the molecular binding does not affect the electronic distribution of the atom so that each constituent atom scatters independently from the others. Put simply, the atoms of the molecule are viewed as isolated entities. Hence, the initial task in any IAM-SCAR computation is to describe the electron scattering from the atoms that make up the molecular target (here, carbon, hydrogen, and oxygen). The electron-atom interaction is represented by an ab initio complex optical potential, where the real part accounts for the elastic scattering dynamics, while the imaginary part represents the inelastic processes. Note that those inelastic processes are considered as "absorption" channels from the incident electron beam. The complex potential for each atom is written as 


$$
\begin{aligned}
V_{\mathrm{opt}}(\boldsymbol{r}) & =V_{\mathrm{R}}(\boldsymbol{r})+i V_{\mathrm{abs}}(\boldsymbol{r}) \\
& =V_{\mathrm{S}}(\boldsymbol{r})+V_{\mathrm{ex}}(\boldsymbol{r})+V_{\mathrm{pol}}(\boldsymbol{r})+i V_{\mathrm{abs}}(\boldsymbol{r}) .
\end{aligned}
$$

In Eq. (2), the real part $(R)$ consists of three terms: (i) a static term $\left(V_{\mathrm{S}}\right)$ derived from a Hartree-Fock calculation of the atomic charge distributions, ${ }^{38}$ (ii) an exchange term $\left(V_{\text {ex }}\right)$ which accounts for the indistinguishability of the incident and target electrons, ${ }^{39}$ and (iii) a polarization potential $\left(V_{\mathrm{pol}}\right)$ that describes the long-range interactions. ${ }^{40}$ Finally, the absorption potential $\left(V_{\mathrm{abs}}\right)$ is based on the quasifree model developed by Staszewska et al. ${ }^{41}$ Initially, quite significant discrepancies between experimental data and the optical potential model were found, although these were subsequently corrected. ${ }^{42,43}$ With those changes, the Spanish team have developed a method capable of describing electron-atom scattering over a broad range of energies and targets (see, e.g., Ref. 44).

The electron-molecule cross sections are now computed from the atomic data using the additivity rule (AR). ${ }^{45}$ Within that approach, the molecular scattering amplitude is derived from the sum of all the relevant atomic amplitudes, including the phase coefficients, from which the molecular DCSs can be determined. ICSs are then derived by integrating those DCSs. The TCSs are finally determined from the sum of the elastic ICSs and the absorption ICSs (for all inelastic processes except rotations and vibrations), at a given energy. The main limitation of this method is that no molecular structure is considered, making it valid only for fast enough incident electrons $\left(\succsim 100 \mathrm{eV}^{43}\right)$ which effectively "see" the target molecule as the sum of the individual atoms. For lower incident energies, the atomic cross sections are sufficiently large to overlap with one another, leading to an overestimation of the AR molecular cross sections. To solve that limitation, at least in part, Blanco and García ${ }^{46,47}$ developed the SCAR code, which takes into account the geometric features of the molecule by introducing some screening coefficients (SC). More recently, they incorporated some interference (I) term corrections. ${ }^{36}$ Within these IAM-SCAR and IAMSCAR+I approaches, the range of the validity of the model might be extended down in energies to $\sim 20-30 \mathrm{eV}$. Certainly, there is evidence to suggest that either the IAM-SCAR or IAM-SCAR+I approaches are quite robust tools for computing cross sections at intermediate to high energies. ${ }^{48-50}$ Note that incorporating the interference term increases the original IAM-SCAR TCS results by $24 \%$ at $10 \mathrm{eV}$ and $27 \%$ at $400 \mathrm{eV}$. All the present IAM-SCAR+I cross sections, for electron scattering from 1-butanol, are summarized in Table I.

In Table I, we also include results from a Born-level calculation for the rotational ICSs (see Ref. 20 for details of this method). While one might instinctively resile from a Born-approximation type result being valid at some of the lower energies we report, it should be remembered that typical rotational-excitation thresholds $(\Delta E)$ of polyatomic molecules are at the $\sim 1 \mathrm{meV}$ level so that even at $E=1 \mathrm{eV}$ we find $E / \Delta E \gg 1$. This latter observation is a criterion that is often cited $^{11}$ for when the Born approximation ${ }^{51}$ might be valid. This is no moot point. Ness et al. ${ }^{52}$ demonstrated that in order to simulate transport coefficients describing electrons moving through a background water gas, under the influence of an applied electric field, at lower regions of the reduced electric field, including rotational ICSs was vital. Similarly, more recently, Casey et al. ${ }^{53}$ demonstrated this

TABLE I. Present IAM-SCAR+I cross sections $\left(\times 10^{-16} \mathrm{~cm}^{2}\right)$ for electron scattering from 1-butanol and rotational ICSs

\begin{tabular}{|c|c|c|c|c|c|c|}
\hline $\mathrm{E}(\mathrm{eV})$ & Elastic ICS & $\mathrm{TICS}^{2}$ & Excitation ICSs & TCS & Rotational ICS & TCS+Rotational ICS \\
\hline 1.0 & 134.12 & 0 & 0 & 134.12 & 115.64 & 249.76 \\
\hline 1.5 & 114.8 & 0 & 0 & 114.8 & 80.64 & 195.44 \\
\hline 2.0 & 102.48 & 0 & 0 & 102.48 & 62.44 & 164.92 \\
\hline 3.0 & 82.6 & 0 & 0 & 82.6 & 43.4 & 126.0 \\
\hline 4.0 & 74.48 & 0 & 0 & 74.48 & 33.6 & 108.08 \\
\hline 5.0 & 71.4 & 0 & 0 & 71.4 & 27.496 & 98.9 \\
\hline 7.0 & 67.2 & 0 & 0 & 67.2 & 20.3 & 87.36 \\
\hline 10 & 63 & 0 & 0.285 & 63.28 & 14.7 & 77.98 \\
\hline 15 & 57.4 & 0.3332 & 2.9708 & 60.76 & 10.16 & 70.92 \\
\hline 20 & 50.4 & 3.528 & 5.208 & 59.08 & 7.81 & 66.89 \\
\hline 30 & 40.88 & 9.968 & 5.236 & 56.0 & 5.40 & 61.40 \\
\hline 40 & 35.28 & 12.684 & 4.396 & 52.36 & 4.14 & 56.50 \\
\hline 50 & 31.92 & 13.58 & 3.948 & 49.28 & 3.39 & 52.67 \\
\hline 70 & 27.16 & 13.72 & 3.5 & 44.24 & 2.48 & 46.72 \\
\hline 100 & 22.848 & 12.852 & 3.108 & 38.92 & 1.78 & 40.70 \\
\hline 150 & 18.592 & 11.144 & 2.716 & 32.48 & 1.23 & 33.71 \\
\hline 200 & 15.932 & 9.744 & 2.492 & 28.28 & 0.94 & 29.22 \\
\hline 300 & 12.544 & 7.84 & 2.1 & 22.484 & 0.644 & 23.128 \\
\hline 400 & 10.472 & 6.524 & 1.848 & 18.844 & 0.4928 & 19.35 \\
\hline 500 & 9.016 & 5.628 & 1.652 & 16.324 & 0.4004 & 16.72 \\
\hline 700 & 7.112 & 4.424 & 1.372 & 12.908 & 0.2912 & 13.19 \\
\hline
\end{tabular}
calculated using the Born approximation. ${ }^{51}$ 
same effect, with tetrahydrofuran now being the background gas. Hence, rotational ICS, such as we report in Table I, will be crucial in simulating electron-transport behavior in 1-butanol.

\section{RESULTS AND DISCUSSION}

The present experimental results of TCSs for electron scattering by 1-butanol, in the energy range from 80 to $400 \mathrm{eV}$, are numerically displayed in Table II. Also listed in Table II is a "hybrid" TCS that has been derived from the elastic ICSs of Khakoo et al. ${ }^{14}$ being added to a Binary-Encounter-Bethe (BEB), with an outer valence Green's function (OVGF) model chemistry, total ionization cross section calculated result from Ghosh et al. ${ }^{2}$ While this means that our "hybrid" TCS ignores the rotational excitation, vibrational excitation, electronic-state excitation, and DEA scattering channels, which, in principle, are all open at the intermediate energies of this investigation, there is plenty of evidence ${ }^{54-56}$ to suggest that the cross sections for those channels will be relatively small in the current energy range. Indeed, considering the rotational ICS of Table I, we find that its contribution to the IAM-SCAR+I TCS drops from $6.4 \%$ at $50 \mathrm{eV}$ to $2.5 \%$ at $400 \mathrm{eV}$. Therefore, it appears to be a priori reasonable to compare our directly measured TCS with the "hybrid" TCS we have derived with that comparison being illustrated in Fig. 2. Also plotted in Fig. 2 are a selection of our IAMSCAR+I cross section results, including the TCS, the experimental TICS from Ghosh et al., and the results from applying two parametric models, describing the energy dependence of the TCS at intermediate to high energies, from García and Manero ${ }^{57}$ and Zecca and colleagues.

Let us now consider Fig. 2 in more detail. It is clear that the qualitative trend, i.e., the behavior of the TCS as a function of energy, in both the present data and the "hybrid" TCS, is very similar. The

TABLE II. Present measured total cross sections $\left(\times 10^{-16} \mathrm{~cm}^{2}\right)$ for electron scattering from 1-butanol. Also included is a "hybrid" TCS, derived using the elastic ICS from Khakoo et al. ${ }^{14}$ and a BEB with OVGF model chemistry total ionization cross section from Ghosh et al. ${ }^{2}$ Errors are the absolute uncertainties on the data, and are at one standard deviation level.

\begin{tabular}{lcc}
\hline \hline $\mathrm{E}(\mathrm{eV})$ & Present TCS & "Hybrid" TCS \\
\hline 1 & $\ldots$ & $118.0 \pm 21.0$ \\
2 & $\ldots$ & $73.1 \pm 13.4$ \\
5 & $\ldots$ & $66.9 \pm 10.9$ \\
10 & $\ldots$ & $60.0 \pm 9.7$ \\
15 & $\ldots$ & $51.9 \pm 7.5$ \\
20 & $\ldots$ & $44.3 \pm 6.6$ \\
30 & $\ldots$ & $54.2 \pm 8.4$ \\
50 & $\ldots$ & $39.8 \pm 6.4$ \\
80 & $52.03 \pm 4.22$ & $\ldots$ \\
100 & $44.32 \pm 2.97$ & $27.9 \pm 4.5$ \\
150 & $37.60 \pm 2.50$ & $\ldots$ \\
200 & $30.47 \pm 2.80$ & $\ldots$ \\
250 & $24.49 \pm 3.20$ & $\ldots$ \\
300 & $20.48 \pm 1.88$ & $\ldots$ \\
350 & $16.73 \pm 0.57$ & $\ldots$ \\
400 & $15.06 \pm 1.63$ & \\
\hline \hline
\end{tabular}

only exception to this is at $30 \mathrm{eV}$, where a structurelike feature appears in the "hybrid" TCS. That feature arises solely from the elastic ICS of Khakoo et al., ${ }^{14}$ which displays a similar result that is not physical but rather is an artefact of their extrapolation of the measured $30 \mathrm{eV} \mathrm{DCS}$ to $0^{\circ}$ and $180^{\circ}$ prior to integration to derive the ICS. Nonetheless, the general behavior in Fig. 2, where the magnitude of the TCS increases as the incident electron energy decreases, is entirely consistent with the polar nature of 1-butanol and that it possesses a dipole polarizability of quite significant magnitude. ${ }^{28}$ There is, however, a mismatch in terms of the magnitudes of their TCSs, with the "hybrid" TCS typically being systematically lower in value compared to that of the present measurement. Interestingly, although we do not explicitly plot this in Fig. 2, the present IAM-SCAR+I elastic ICS and the elastic ICS from Khakoo et al., ${ }^{14}$ over the common energy range $1-100 \mathrm{eV}$, are typically, to within the cited uncertainties on the Khakoo et al. results, in very good agreement with the notable exceptions being at $30 \mathrm{eV}$ where the Khakoo et al. ICS is a little too high and $100 \mathrm{eV}$ where the Khakoo et al. result is lower in magnitude. This latter observation is a little counterintuitive, as the IAM-SCAR+I result would be expected to perform better at higher energies which, therefore, suggests a problem with the Khakoo et al. elastic ICS result at $100 \mathrm{eV}$. However, we do not believe that this observation indicates any short comings with the measured DCS of Khakoo et al., ${ }^{14}$ rather, it reflects the difficulty in extrapolating DCS measured over a finite scattered electron angular range to $0^{\circ}$ and $180^{\circ}$ before integrating. As the $100 \mathrm{eV}$ elastic ICS of Khakoo et al. is too low in magnitude, this also carries through to the "hybrid" TCS being somewhat lower in magnitude at $100 \mathrm{eV}$. In addition, of course, the fact that our "hybrid" TCS is ignoring open scattering channels such as rotational, vibrational, electronic-state, DEA, and neutral dissociation must also contribute to the discrepancy in magnitude that we observe in Fig. 2. If, again at $100 \mathrm{eV}$, we were to add from Table I the IAM-SCAR + I result for the sum over all inelastic channels (except rotations and vibrations) to the Born ICS for rotational excitation, to the "hybrid" TCS in Table II, then the level of accord found with our measured TCS improves quite a bit. This observation suggests that those scattering channels are contributing relatively more to the TCS for 1-butanol than we have found previously for other targets. $^{54-56}$

We can now compare in Fig. 2 our IAM-SCAR+I TCS to our measured TCS. It is apparent that generally a fair accord, in terms of both the energy dependence of the TCS and its magnitude, is found between them. However, the level of accord seen in Fig. 2 for 1-butanol is not as impressive as that found previously for 1-propanol. ${ }^{25}$ This suggests that our screening and interference correction terms for 1-butanol, to the IAM method, have not been as effective here as they were in 1-propanol. ${ }^{25}$ Also plotted in Fig. 2 are TCS results from the parametric models of García and Manero ${ }^{57}$ and Zecca and colleagues. ${ }^{58,59}$ In the former case [see their Eq. (5)], García and Manero parameterize the TCS as a function of energy in terms of $\alpha$ (dipole polarizability) and $Z$ (atomic number). It is apparent from Fig. 2, however, that while their approach ${ }^{57}$ does a very good job in reproducing the shape of the TCS, it underestimates its magnitude over all energies. On the other hand, the two parameter $\left(\sigma_{0}\right.$ and $\left.B\right)$ fit of the model of Zecca and colleagues ${ }^{58,59}$ reproduces very well the present TCS. Nonetheless, it must be noted that $\sigma_{0}$ and $B$ have no real physical meaning, and they are simply 


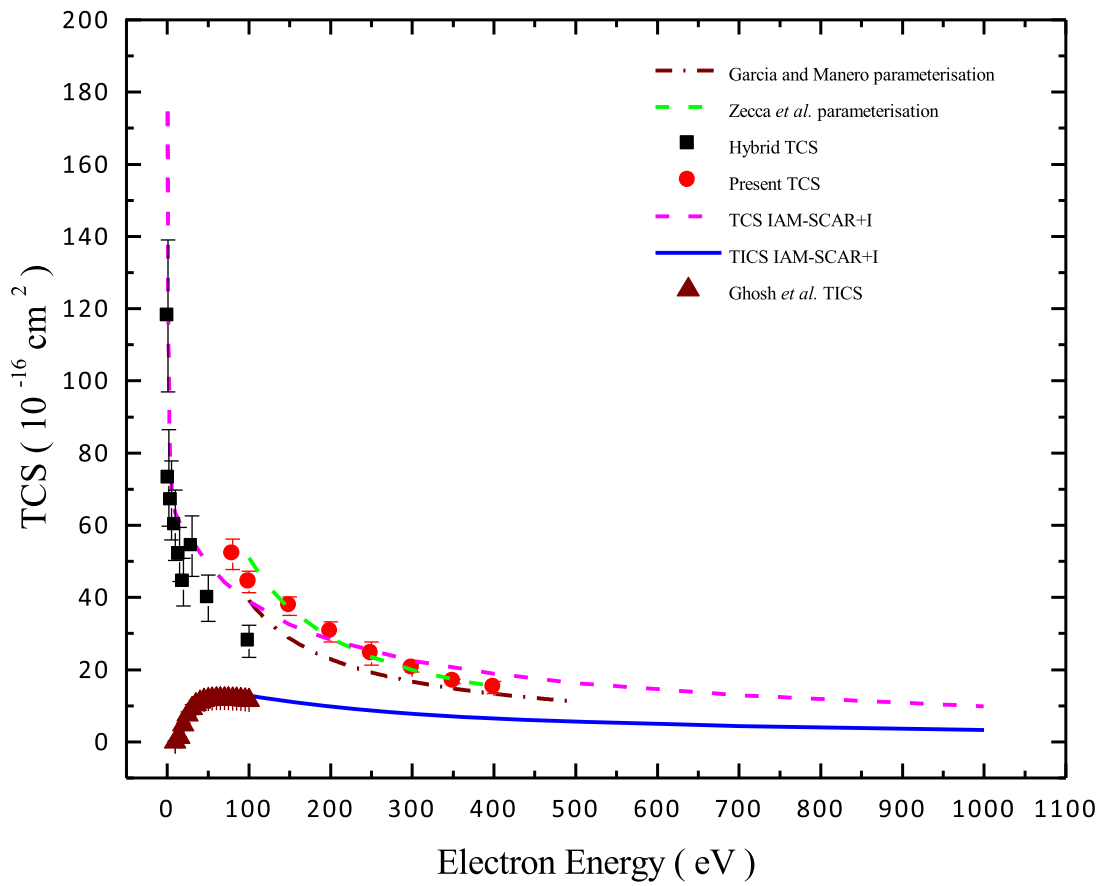

FIG. 2. Plot showing a selection of the electron-1-butanol scattering cross sections $\left(\times 10^{-16} \mathrm{~cm}^{2}\right)$. The present measured TCS (red filled circle), a "hybrid" TCS (black filled square), and the TCS from our IAM-SCAR+I calculations (pink dashed line) are plotted. Also shown are TCS results using the parametric forms of García and Manero ${ }^{57}$ (brown dashed-dotted line) and Zecca and colleagues ${ }^{5,59}$ (green dashed line). The IAM-SCAR+I TICS (blue solid line) and measured TICS data from Ghosh et al. ${ }^{2}$ (brown filled triangle) are additionally plotted.

fitting parameters so that the observed level of agreement is not surprising. Indeed, all we can really say about the intermediate energy parameterization of Zecca and colleagues is that it represents well the energy dependence of the measured TCS in 1-butanol, as it did previously for many other molecules. ${ }^{58,59}$

In terms of constructing a cross section database, for modeling or simulation studies in 1-butanol, then the results embodied in Fig. 2 are quite encouraging. First, we see that the measured TICS from Ghosh et al. ${ }^{2}$ and our IAM-SCAR+I TICS are in good accord. Second, we also find that over the common energy range, the present experimental TCS and our calculated IAM-SCAR+I TCS are in pretty good accord. As noted earlier, although not explicitly shown, the agreement between our elastic IAM-SCAR+I ICS and the corresponding measured elastic ICS from Khakoo et al. ${ }^{14}$ is also very good. Given that IAM-SCAR+I computations are providing good estimates for the elastic, ionization, and total cross sections, it is reasonable to infer that their values for the sum over all inelastic integral cross sections are also realistic. Hence, it would appear that the makings of a cross section database for simulating electron transport in 1-butanol is coming together. Of course, it would be highly desirable if those inelastic processes could be separated out, with the measurement of absolute DEA cross sections and electronicstate cross sections being important. Similarly, specific measurements or calculations for vibrational-excitation ICS would be crucial $^{60}$ before any complete 1-butanol database could be assembled. In addition, extensions to lower energies where resonance effects may be important ${ }^{16,17}$ are also needed.

Finally, let us now consider Fig. 3 which shows all the UFJF total cross section results for electron scattering from the linear primary alcohols methanol, ${ }^{24}$ ethanol, ${ }^{24} 1$-propanol, ${ }^{25}$ and 1-butanol. It is immediately apparent from Fig. 3 that as the length of the primary alcohol chain increases, at any given incident electron energy, the TCS increases. We had previously noted in our Introduction that $\alpha_{1 \text {-butanol }}>\alpha_{1 \text {-propanol }}>\alpha_{\text {ethanol }}>\alpha_{\text {methanol }}$. As the dipole polarizability in some sense measures the spatial extent of the target molecular electron cloud, the trends in Fig. 3 make good qualitative sense. Namely, the bigger the molecular charge cloud, the more likely the incident electron will interact with it, leading to scattering and, therefore, the larger the TCS will be. So in this sense, the results in Fig. 3 are almost classical in nature in that the larger is the target you are aiming at the more likely it is that you will hit it! To further probe this notion about the role of the dipole polarizability on the electron scattering dynamics of the primary alcohols, in Fig. 3, we have also plotted curves for ethanol, 1-propanol, and 1-butanol that are obtained from the relationship

$$
\operatorname{TCS}^{i}(E)=\frac{\alpha^{i}}{\alpha_{\text {methanol }}} \operatorname{TCS}_{\text {methanol }}(E)
$$

where $i=$ ethanol, 1-propanol, and 1-butanol, $\alpha$ are the respective dipole polarizabilities, ${ }^{28}$ and the $\operatorname{TCS}_{\text {methanol }}(E)$ is taken from Silva et $a l .{ }^{24}$ Note that we have done this for $E \gtrsim 70 \mathrm{eV}$. Equation (3) affords a good qualitative description of the respective ethanol, 1-propanol, and 1-butanol TCSs. Indeed for the ethanol TCS, Eq. (3) actually provides a good quantitative description of that cross section. However, particularly as you approach $70 \mathrm{eV}$, there is a noticeable difference in magnitude between the measured TCS $^{24,25}$ of 1propanol and 1-butanol and the data generated using Eq. (3). There has been quite a lot of work in the positron scattering community, ${ }^{33}$ at the TCS level, particularly from the Yamaguchi ${ }^{61}$ and Trento groups, ${ }^{62-64}$ in comparing the positron and electron scattering TCS for a given molecule. That work invariably found that at some energy 


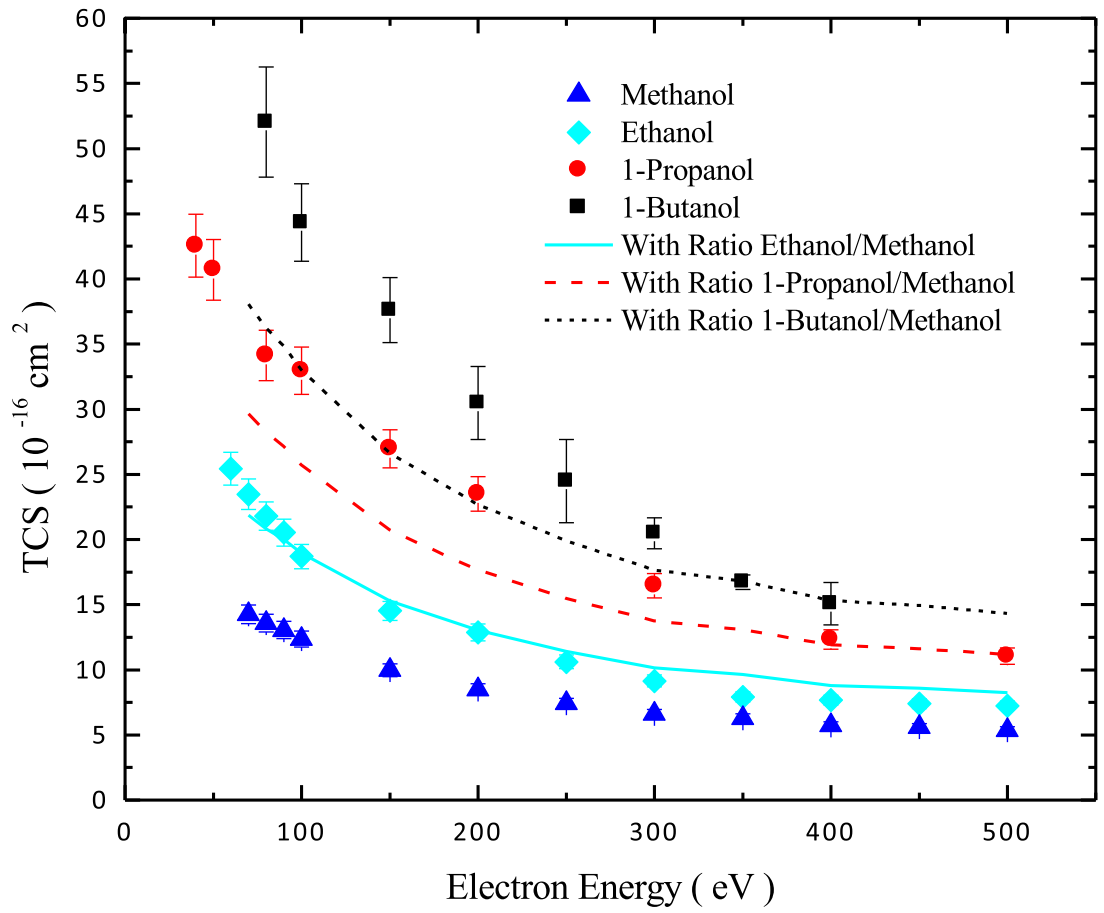

FIG. 3. Comparison of TCS $\left(\times 10^{-16} \mathrm{~cm}^{2}\right)$ measured at UFJF ${ }^{24}$ for methanol (blue filled triangle), ethanol (turquoise filled diamond), 1-propanol (red filled circle), and 1-butanol (black filled square). Also plotted are data we have derived from Eq. (3), for ethanol (turquoise solid line), 1-propanol (red dashed line), and 1-butanol (black dashed line). See text for further details.

the TCS for the two leptons merged. The rationale behind that observation was that above this energy both the exchange interaction (in the case of incident electrons) and the positronium formation interaction (in the case of incident positrons) became negligible. The actual value of energy where the positron and electron TCSs merged was molecule specific, but it was always greater than $100 \mathrm{eV}$. We believe that, at least in part, one of the reasons Eq. (3) fails to reproduce the TCS for 1-propanol and 1-butanol is simply that the exchange interaction is still playing an important role in their scattering dynamics above $70 \mathrm{eV}$ and up to about $350 \mathrm{eV}$. The fact that Eq. (3) does provide a good description of the measured TCS for 1-propanol and 1-butanol above $350 \mathrm{eV}$, where electron exchange is expected to be a very small effect, supports our assertion here. As Eq. (3) does not account for electron exchange, we see that it underestimates the measured TCS magnitudes with the extent of that underestimation increasing as you approach $70 \mathrm{eV}$. Furthermore, the extent of the disagreement at a given energy below $350 \mathrm{eV}$ increases as you go from 1-propanol to 1-butanol. This latter observation also supports our hypothesis because 1-butanol contains more target electrons to exchange with the target projectile, compared to 1-propanol, so that if exchange were an important consideration, here you would anticipate its effect to be stronger in 1-butanol than 1-propanol as we observe (see Fig. 3). Of course, the polar nature of the primary alcohols must also be playing a role in what we observe in Fig. 3. While we had previously noted that the values of $\mu$ for methanol, ethanol, 1-propanol and 1-butanol all lie in a narrow range between 1.55 and $1.70 \mathrm{D}$, they are indeed a little different in each case so that it is reasonable to expect that their influence on the scattering dynamics will also be somewhat different in each case.

\section{CONCLUSIONS}

We have reported results on total cross section measurements and IAM-SCAR $+\mathrm{I}$ computations for electron scattering from 1-butanol. In addition, IAM-SCAR+I results for the elastic ICS, sum over all inelastic ICS and TICS, ${ }^{2}$ and a Born cross section for rotational excitation have been reported. Both the theoretical and experimental TCS exhibited the property that the magnitude of the TCS increased as the incident electron energy decreased, a behavior consistent with the important role played by the target molecule physicochemical properties, such as the dipole moment and dipole polarizability, in the scattering process. By considering the measured TCS results, for methanol, ethanol, 1-propanol, and 1-butanol, ${ }^{24,25}$ from UFJF, we were also able to tie down the very important role of the dipole polarizability in their electron scattering dynamics. However, contributions to their TCS due to electron exchange and the permanent dipole moments of these polar species were also apparent from considering those TCS trends.

While the cross sections presented in this paper, and those from Ghosh et al. ${ }^{2}$ and Khakoo et al., ${ }^{14}$ go part of the way toward realizing a database that fulfills the criteria of Tanaka et al. ${ }^{11}$ for simulation and modeling studies, further experimental and theoretical work is clearly required. In particular, specific measurements and/or calculations for vibrational excitation and discrete electronic-state excitation, as well as absolute DEA data, at energies approaching threshold as well as at intermediate energies, are required. Nonetheless, the database as it currently stands should permit some preliminary electron transport studies, in the linear primary alcohols, to be undertaken. 


\section{ACKNOWLEDGMENTS}

M.C.A.L. acknowledges financial support from the Brazilian Conselho Nacional de Desenvolvimento Cientifico e Tecnológico (CNPq), Fundação de Amparo à Pesquisa do Estado de Minas Gerais (FAPEMIG), and FINEP, while M.).B. thanks CNPq for his award to enable him to visit UFJF. Some financial assistance from the Australian Research Council through Grant Nos. DP160102787 and DP180101655 is also noted. S. Ghosh acknowledges his grant from PNPD/CAPES, while G.G. thanks the Spanish Ministerio de Economia, Industria y Competitividad for his project Grant No. FIS2016-80440 and the EU Project No. FP7-ITN-ARGENT608163 .

\section{REFERENCES}

${ }^{1}$ W. A. D. Pires, K. L. Nixon, S. Ghosh, R. A. A. Amorin, R. F. C. Neves, H. V. Duque, D. G. M. da Silva, D. B. Jones, M. J. Brunger, and M. C. A. Lopes, Int. J. Mass Spectrom. 430, 158 (2018).

${ }^{2}$ S. Ghosh, K. L. Nixon, W. A. D. Pires, R. A. A. Amorin, R. F. C. Neves, H. V. Duque, D. G. M. da Silva, D. B. Jones, F. Blanco, G. Garcia, M. J. Brunger, and M. C. A. Lopes, Int. J. Mass Spectrom. 430, 44 (2018).

${ }^{3}$ See http://biofuel.org.uk for Biofuels, the fuel of the future for details on some properties of 1-butanol.

${ }^{4}$ L. Campbell and M. J. Brunger, Plasma Sources Sci. Technol. 22, 013002 (2013).

${ }^{5}$ L. Campbell and M. J. Brunger, Int. Rev. Phys. Chem. 35, 297 (2016).

${ }^{6}$ M. A. Ridenti, J. Amorim Filho, M. J. Brunger, R. F. da Costa, M. T. do N. Varella, M. H. F. Bettega, and M. A. P. Lima, Eur. Phys. J. D 70, 161 (2016).

${ }^{7}$ A. G. Sanz, M. C. Fuss, A. Muñoz, F. Blanco, P. Limão-Vieira, M. J. Brunger, S. J. Buckman, and G. García, Int. J. Radiat. Biol. 88, 71 (2012).

${ }^{8}$ M. C. Fuss, L. Ellis-Gibbings, D. B. Jones, M. J. Brunger, F. Blanco, A. Muñoz, P. Limão-Vieira, and G. García, J. Appl. Phys. 117, 214701 (2015).

${ }^{9}$ N. A. Garland, M. J. Brunger, G. Garcia, J. de Urquijo, and R. D. White, Phys. Rev. A 88, 062712 (2013).

${ }^{10}$ R. D. White, M. J. Brunger, N. A. Garland, R. E. Robson, K. F. Ness, G. Garcia, J. de Urquijo, S. Dujko, and Z. Lj. Petrović, Eur. Phys. J. D 68, 125 (2014).

${ }^{11}$ H. Tanaka, M. J. Brunger, L. Campbell, H. Kato, M. Hoshino, and A. R. P. Rau, Rev. Mod. Phys. 88, 025004 (2016).

${ }^{12}$ L. C. Pitchford, L. L. Alves, K. Bartschat, S. F. Biagi, M.-C. Bordage, I. Bray, C. E. Brion, M. J. Brunger, L. Campbell et al., Plasma Processes Polym. 14, 1600098 (2017).

${ }^{13}$ J. E. Hudson, M. L. Hamilton, C. Vallance, and P. W. Harland, Phys. Chem. Chem. Phys. 5, 3162 (2003).

${ }^{14}$ M. A. Khakoo, J. Muse, H. Silva, M. C. A. Lopes, C. Winstead, V. McKoy, E. M. de Oliveira, R. F. da Costa, M. T. do N. Varella, M. H. F. Bettega, and M. A. P. Lima, Phys. Rev. A 78, 062714 (2008).

${ }^{15}$ B. C. Ibănescu and M. Allan, Phys. Chem. Chem. Phys. 11, 7640 (2009).

${ }^{16}$ M. J. Brunger and S. J. Buckman, Phys. Rep. 357, 215 (2002).

${ }^{17}$ M. J. Brunger, Int. Rev. Phys. Chem. 36, 333 (2017).

${ }^{18}$ D. V. Fursa, M. C. Zammit, R. L. Threlfall, J. S. Savage, and I. Bray, Phys. Rev. A 96, 022709 (2017).

${ }^{19}$ M. C. Fuss, A. G. Sanz, F. Blanco, J. C. Oller, P. Limão-Vieira, M. J. Brunger, and G. García, Phys. Rev. A 88, 042702 (2013).

${ }^{20}$ A. Traoré Dubuis, A. Verkhovtsev, L. Ellis-Gibbings, K. Krupa, F. Blanco, D. B. Jones, M. J. Brunger, and G. García, J. Chem. Phys. 147, 054301 (2017).

${ }^{21}$ J. D. Gorfinkiel and S. Ptasinska, J. Phys. B: At., Mol. Opt. Phys. 50, 182001 (2017).

${ }^{22}$ R. F. da Costa, E. M. de Oliveira, M. H. F. Bettega, M. T. do N. Varella, D. B. Jones, M. J. Brunger, F. Blanco, R. Colmenares, P. Limão-Vieira, G. García, and M. A. P. Lima, J. Chem. Phys. 142, 104304 (2015).

${ }^{23}$ R. F. da Costa, M. T. do N. Varella, M. H. F. Bettega, R. F. C. Neves, M. C. A. Lopes, F. Blanco, G. García, D. B. Jones, M. J. Brunger, and M. A. P. Lima, J. Chem. Phys. 144, 124310 (2016).
${ }^{24}$ D. G. M. Silva, T. Tejo, J. Muse, D. Romeo, M. A. Khakoo, and M. C. A. Lopes, J. Phys. B: At., Mol. Opt. Phys. 43, 015201 (2010).

${ }^{25}$ D. G. M. da Silva, M. Gomes, S. Ghosh, I. F. L. Silva, W. A. D. Pires, D. B. Jones, F. Blanco, G. Garcia, S. J. Buckman, M. J. Brunger, and M. C. A. Lopes, J. Chem. Phys. 147, 194307 (2017).

${ }^{26}$ CRC Handbook of Chemistry and Physics, 79th ed., edited by D. R. Lide (CRC Press, Boca Raton, 1998).

${ }^{27}$ NIST Standard Reference Data at URL: http://srdata.nist.gov/cccbdb for tabulated values of dipole moments.

${ }^{28}$ M. Gussoni, M. Rui, and G. Zerbi, J. Mol. Struct. 447, 163 (1998).

${ }^{29}$ R. W. Wagenaar and F. J. de Heer, J. Phys. B: At., Mol. Opt. Phys. 18, 2021 (1985).

${ }^{30}$ C. Szmytkowski, K. Maciag, and G. Karwasz, Phys. Scr. 54, 271 (1996).

${ }^{31} \mathrm{See} \quad$ https://www.mksinst.com/Docs/R/GP-203LeakValve-203026-MAN.pdf for manufacturer's specifications.

${ }^{32}$ See http:webbook.nist.gov/cgi/inchi?ID=C712388Mask=4\& Type=ANTOINE\& Plot=on for the Antoine equation.

${ }^{33}$ M. J. Brunger, S. J. Buckman, and K. Ratnavelu, J. Phys. Chem. Ref. Data 46, 023102 (2017).

${ }^{34}$ A. G. Sanz, M. C. Fuss, F. Blanco, J. D. Gorfinkiel, D. Almeida, F. F. da Silva, P. Limão-Vieira, M. J. Brunger, and G. García, J. Chem. Phys. 139, 184310 (2013).

${ }^{35}$ F. Blanco, J. Rosada, A. Illana, and G. García, Phys. Lett. A 374, 4420 (2010).

${ }^{36}$ F. Blanco, L. Ellis-Gibbings, and G. García, Chem. Phys. Lett. 645, 71 (2016).

${ }^{37}$ F. Blanco and G. Garcia, J. Phys. B: At., Mol. Opt. Phys. 42, 145203 (2009).

${ }^{38} \mathrm{R}$. D. Cowan, The Theory of Atomic Structure and Spectra (University of California Press, London, 1981).

${ }^{39}$ M. E. Riley and D. G. Truhlar, J. Chem. Phys. 63, 2182 (1975).

${ }^{40}$ X. Zhang, J. Sun, and Y. Liu, J. Phys. B: At., Mol. Opt. Phys. 25, 1893 (1992).

${ }^{41}$ G. Staszewska, D. W. Schwenke, D. Thirumalai, and D. G. Truhlar, Phys. Rev. A 28, 2740 (1983).

${ }^{42}$ F. Blanco and G. García, Phys. Lett. A 295, 178 (2002).

${ }^{43}$ F. Blanco and G. García, Phys. Rev. A 67, 022701 (2003).

${ }^{44}$ O. Zatsarinny, K. Bartschat, G. Garcia, F. Blanco, L. R. Hargreaves, D. B. Jones, R. Murrie, J. R. Brunton, M. J. Brunger, M. Hoshino, and S. J. Buckman, Phys. Rev. A 83, 042702 (2011).

${ }^{45}$ Y. Jiang, J. Sun, and L. Wan, Phys. Rev. A 52, 398 (1995).

${ }^{46}$ F. Blanco and G. García, Phys. Lett. A 317, 458 (2003).

${ }^{47}$ F. Blanco and G. García, Phys. Lett. A 330, 230 (2004).

${ }^{48}$ M. Fuss, A. Muñoz, J. C. Oller, F. Blanco, D. Almeida, P. Limão-Vieira, T. P. D. Do, M. J. Brunger, and G. García, Phys. Rev. A 80, 052709 (2009).

${ }^{49}$ L. R. Hargreaves, J. R. Brunton, A. Prajapati, M. Hoshino, F. Blanco, G. García, S. J. Buckman, and M. J. Brunger, J. Phys. B: At., Mol. Opt. Phys. 44, 045207 (2011).

${ }^{50}$ A. I. Lozano, K. Krupa, F. Ferreira da Silva, P. Limão-Vieira, F. Blanco, A. Muñoz, D. B. Jones, M. J. Brunger, and G. Garcia, Eur. Phys. J. D 71, 226 (2017).

${ }^{51}$ A. Jain, J. Phys. B: At., Mol. Opt. Phys. 21, 905 (1988).

${ }^{52}$ K. F. Ness, R. E. Robson, M. J. Brunger, and R. D. White, J. Chem. Phys. 136, 024318 (2012).

${ }^{53}$ M. J. E. Casey, J. de Urquijo, L. N. Serkovic Loli, D. G. Cocks, G. J. Boyle, D. B. Jones, M. J. Brunger, and R. D. White, J. Chem. Phys. 147, 195103 (2017).

${ }^{54}$ D. B. Jones, L. Ellis-Gibbings, G. García, K. L. Nixon, M. C. A. Lopes, and M. J. Brunger, J. Chem. Phys. 143, 094304 (2015).

${ }^{55}$ R. F. C. Neves, D. B. Jones, M. C. A. Lopes, F. Blanco, G. García, K. Ratnavelu, and M. J. Brunger, J. Chem. Phys. 142, 194305 (2015).

${ }^{56}$ D. B. Jones, R. F. da Costa, M. T. do N. Varello, M. H. F. Bettega, M. A. P. Lima, F. Blanco, G. García, and M. J. Brunger, J. Chem. Phys. 144, 144303 (2016).

${ }^{57}$ G. García and F. Manero, Chem. Phys. Lett. 280, 419 (1997).

${ }^{58}$ A. Zecca, G. P. Karwasz, and R. S. Brusa, Phys. Rev. A 45, 2777 (1992).

${ }^{59}$ P. Możejko, G. Kasperski, C. Szmytkowski, G. P. Karwasz, R. S. Brusa, and A. Zecca, Chem. Phys. Lett. 257, 309 (1996). 
${ }^{60}$ M. J. Brunger, K. Ratnavelu, S. J. Buckman, D. B. Jones, A. Muñoz, F. Blanco, and G. Garcia, Eur. Phys. J. D 70, 46 (2016).

${ }^{61}$ M. Kimura, O. Sueoka, A. Hamada, and Y. Itikawa, Adv. Chem. Phys. 111, 537 (2000).

${ }^{62}$ A. Zecca, L. Chiari, G. Garcia, F. Blanco, E. Trainotti, and M. J. Brunger, J. Phys. B: At., Mol. Opt. Phys. 43, 215204 (2010).
${ }^{63}$ L. Chiari, A. Zecca, E. Trainotti, M. H. F. Bettega, S. d'A Sanchez, M. T. do N. Varella, M. A. P. Lima, and M. J. Brunger, J. Phys. B: At., Mol. Opt. Phys. 44, $195202(2011)$

${ }^{64}$ M. T. do N. Varella, S. d'A. Sanchez, M. H. F. Bettega, M. A. P. Lima, L. Chiari, A. Zecca, E. Trainotti, and M. J. Brunger, J. Phys. B: At., Mol. Opt. Phys. 46, 175202 (2013). 